Draft version March 25, 2020

Preprint typeset using $\mathrm{IAT}_{\mathrm{E}} \mathrm{X}$ style emulateapj v. 12/16/11

\title{
GASEOUS DYNAMICAL FRICTION IN PRESENCE OF BLACK HOLE RADIATIVE FEEDBACK
}

\author{
KWANGHo PARK AND TAMara Bogdanović \\ Center for Relativistic Astrophysics, School of Physics, Georgia Institute of Technology, Atlanta, GA 30332, USA \\ Draft version March 25, 2020
}

\begin{abstract}
Dynamical friction is thought to be a principal mechanism responsible for orbital evolution of massive black holes (MBHs) in the aftermath of galactic mergers and an important channel for formation of gravitationally bound $\mathrm{MBH}$ binaries. We use $2 \mathrm{D}$ radiative hydrodynamic simulations to investigate the efficiency of dynamical friction in the presence of radiative feedback from an $\mathrm{MBH}$ moving through a uniform density gas. We find that ionizing radiation that emerges from the innermost parts of the MBH's accretion flow strongly affects the dynamical friction wake and renders dynamical friction inefficient for a range of physical scenarios. MBHs in this regime tend to experience positive net acceleration, meaning that they speed up, contrary to the expectations for gaseous dynamical friction in absence of radiative feedback. The magnitude of this acceleration is however negligibly small and should not significantly alter the velocity of $\mathrm{MBHs}$ over relevant physical timescales. Our results suggest that suppression of dynamical friction is more severe at the lower mass end of the $\mathrm{MBH}$ spectrum which, compounded with inefficiency of the gas drag for lower mass objects in general, implies that $<10^{7} M_{\odot}$ MBHs have fewer means to reach the centers of merged galaxies. These findings provide formulation for a sub-resolution model of dynamical friction in presence of $\mathrm{MBH}$ radiative feedback that can be easily implemented in large scale simulations.
\end{abstract}

Subject headings: accretion, accretion disks - black hole physics - hydrodynamics - radiative transfer

\section{INTRODUCTION}

A massive perturber moving through some background medium creates in it a density wake. The wake trails the perturber and exerts on it a gravitational force in the direction opposite to the motion, thus acting as a brake and earning this interaction a name: "dynamical friction." In his seminal work, Chandrasekhar (1943) studied the efficiency of dynamical friction for a massive perturber moving through a stellar background and found the drag force to be maximized when the velocity of the perturber is comparable to the velocity dispersion of stars, $v \approx \sigma$. Ostriker (1999) later evaluated the dynamical friction force acting on a perturber traveling through a uniform gaseous medium and found that the gaseous drag is more efficient than stellar drag for transonic perturbers. This work also established that the gaseous drag is operating most efficiently when $1<\mathcal{M} \lesssim$ few, where the Mach number $\mathcal{M} \equiv v / c_{s, \infty}$ is defined as the ratio between the perturber velocity $v$ and the sound speed of the ambient gas "at infinity" $c_{s, \infty}$, i.e., in a distant region unaffected by the perturber. In this mildly supersonic regime, the dynamical friction force takes form

$$
F_{\mathrm{DF}}=-\frac{4 \pi\left(G M_{\mathrm{BH}}\right)^{2} \rho_{\infty}}{v^{2}} \ln \left[\Lambda\left(1-\frac{1}{\mathcal{M}^{2}}\right)^{0.5}\right]
$$

where $M_{\mathrm{BH}}$ is the mass of the perturber (hereafter assumed to be a black hole; $\mathrm{BH})$ and $\rho_{\infty}$ is the density of the gas at infinity. $\ln \Lambda=\ln \left(r_{\max } / r_{\min }\right)$ is the Coulomb logarithm and $r_{\min }$ and $r_{\max }$ represent the smallest and largest spatial scales in the gas wake that contribute to dynamical friction, respectively. Based on Equation (1), it follows that the effect of dynamical friction is expected

kwangho.park@physics.gatech.edu,tamarab@gatech.edu to be stronger for massive perturbers given that they can interact with a sufficiently dense pool of ambient gas.

Because of its efficiency, gaseous drag has been extensively investigated in simulations that follow pairing of massive black holes (MBHs) in gas rich mergers of galaxies (see Mayer 2013, for review). The most important questions for this research area are: in which galaxies does gaseous dynamical friction lead to successful gravitational pairing of MBHs and on what timescales? Callegari et al. $(2009,2011)$, for example, find that MBH pairs with mass ratios $q<0.1$ are less likely to form gravitationally bound binaries within a Hubble time, largely due to the inefficiency of the gas drag on the smaller of the two MBHs. When pairing is successful, gaseous dynamical friction is capable of transporting the MBHs from galactic radii of a few hundred to $\sim 1$ pc on timescales as short as $\sim 10^{7}$ year and tens of times faster than stellar dynamical friction (for e.g., Escala et al. 2005; Dotti et al. 2006; Mayer et al. 2007).

Interestingly, gravitational interaction of the $\mathrm{MBH}$ with the surrounding gas is quite local. For example, for a $\mathrm{BH}$ with mass $\sim 10^{6} M_{\odot}$ most of the gravitational drag force is contributed by the gas that resides within only a few parsecs of the MBH (Chapon et al. 2013). This proximity implies that the dynamical friction wake can be strongly affected, and possibly obliterated, by irradiation and feedback from an accreting $\mathrm{MBH}$. Indeed, studies of dynamical evolution of MBHs find that dynamical friction can be significantly reduced due to the wake dispersal caused by purely thermal feedback from MBHs in simulations that follow gravitationally recoiled MBHs (Sijacki et al. 2011) and BH pairing (Souza Lima et al. 2016) where the term "wake evacuation" is dubbed. These results bring into question the assumed efficacy of gaseous dynamical friction and call for further exploration of the effects of radiative feedback powered by 
$\mathrm{MBH}$ accretion.

Here, we investigate this question by considering interaction of matter and radiation in the gravitational potential well of a moving $\mathrm{MBH}$. The most important findings of this work are that there are regimes, set by the properties of the $\mathrm{MBH}$ and its ambient medium, in which gas dynamical friction is rendered inefficient by the $\mathrm{MBH}$ feedback. We lay out the relevant physical regimes and scales in Section 2 and evaluate the efficiency of dynamical friction using local radiation hydrodynamic simulations in Section 3. We discuss implications of our findings and conclude in Section 4.

\section{REGIMES OF DYNAMICAL FRICTION IN THE PRESENCE OF RADIATIVE FEEDBACK}

Photoionization and radiation pressure exerted by radiation escaping from the innermost parts of the $\mathrm{BH}$ accretion flow can create the ionized region (the Strömgren sphere or H II region) around the a BH and strongly alter the properties of surrounding gas. This radiation "response", also referred to as the $\mathrm{BH}$ radiative feedback, has been found to lower the accretion rate by orders of magnitude relative to a fiducial case in which radiative feedback is neglected (Milosavljević et al. 2009; Li 2011; Park \& Ricotti 2011, 2012; Pacucci \& Ferrara 2015; Park et al. 2016). In the context of dynamical friction, this accretion regime is also likely to correspond to reduced efficiency of the gas drag due to the impact of ionizing radiation on the BH's density wake.

Park et al. (2014a) and Inayoshi et al. (2016) however find a physical regime at higher gas densities in which the nature of accretion onto the $\mathrm{BH}$ fundamentally changes from this picture, as the H II region collapses under the gravity of the surrounding gas, and accretion continues unaffected by radiation at supper Eddington rates. The two studies differ in their treatment of radiation transport: the latter considers the effects of radiation trapping at high accretion rates (Begelman 1978, 1979) while the former neglects them. Both nevertheless indicate that conditions for hyper-accretion arise for a $\mathrm{BH}$ immersed in neutral gas with number density $n_{\infty}$, when $n_{\infty} M_{\mathrm{BH}}>10^{9}-10^{10} M_{\odot} \mathrm{cm}^{-3}$. This finding implies that the density distribution of gas in the hyper-accretion regime is only weakly affected by radiation pressure, and thus the efficiency of dynamical friction is likely to be restored to that predicted classically, i.e., in absence of radiative feedback.

For moving BHs, accretion rate, and thus accretion luminosity, also depend on $\mathrm{BH}$ velocity. We draw on the results of Park \& Ricotti (2013, hereafter PR13), who used radiation hydrodynamic simulations to investigate the growth and luminosity of BHs moving through a uniform gaseous medium with temperature $T_{\infty}=10^{4} \mathrm{~K}$. PR13 show that in this case the $\mathrm{BH}$ radiative feedback causes a formation of an $\mathrm{H}$ II region elongated along the direction of the $\mathrm{BH}$ motion and filled with ionized hydrogen (or $\mathrm{H}-\mathrm{He}$ ) gas of temperature, $T_{\text {in }} \approx 4 \times 10^{4} \mathrm{~K}$ $\left(6 \times 10^{4} \mathrm{~K}\right)$. A shell of gas with increased density, corresponding to $\left(1+\mathcal{M}^{2}\right) n_{\infty}$, forms in front of the $\mathrm{BH}$ as a consequence of the "snowplow" effect caused by radiation pressure. For a wide range of simulated gas densities the shell becomes gravitationally unstable and collapses when $\mathcal{M} \gtrsim 4$, restoring the properties of the gas flow around the $\mathrm{BH}$ to the classical Bondi-Hoyle-Lyttleton solution (Hoyle \& Lyttleton 1939; Bondi \& Hoyle 1944). While PR13 have not explicitly investigated the properties of the gas wake in their simulations, it follows that in this regime the efficiency of dynamical friction is restored to its classically derived value.

We therefore focus on scenarios described by $1 \leq \mathcal{M}<$ 4 , when gas dynamical friction is classically expected to be most efficient, and $\left(1+\mathcal{M}^{2}\right) M_{\mathrm{BH}} n_{\infty}<10^{9} M_{\odot} \mathrm{cm}^{-3}$, when the influence of the $\mathrm{BH}$ radiative feedback on dynamical friction wake is expected to be significant. Having established the relevant regime we estimate the extent of the dynamical friction wake and compare it to the size of the $\mathrm{H}$ II region.

\subsection{Relevant scales}

The extent of the dynamical friction wake that would form behind the $\mathrm{MBH}$ in absence of radiative feedback can be estimated as a radius of the gravitational influence of the $\mathrm{MBH}$

$$
R_{\mathrm{DF}} \approx \frac{G M_{\mathrm{BH}}}{c_{\mathrm{s}, \infty}^{2}}=52.1 \mathrm{pc}\left(\frac{T_{\infty}}{10^{4} \mathrm{~K}}\right)^{-1}\left(\frac{M_{\mathrm{BH}}}{10^{6} M_{\odot}}\right) .
$$

Here $c_{s, \infty}=\sqrt{\gamma k_{\mathrm{B}} T_{\infty} / \mu m_{p}}=9.1 \mathrm{~km} \mathrm{~s}^{-1}\left(T_{\infty} / 10^{4} \mathrm{~K}\right)^{1 / 2}$, assuming isothermal gas with hydrogen composition characterized by the adiabatic index $\gamma=1$ and mean atomic weight $\mu=1$. The constants have their usual meaning. As mentioned in Section 1, most of the gravitational drag force is contributed by the gas that resides within an order of magnitude smaller region than that estimated in equation (2), so this value can be considered a conservative upper limit.

The size of the elongated HII region sensitively depends on the $\mathrm{MBH}$ accretion rate and velocity. For the purpose of the estimates presented here, we neglect the elongation of the $\mathrm{H}$ II region and estimate the radius of a spherically symmetric ionization sphere that would form around a stationary $\mathrm{MBH}$ accreting hydrogen gas, $R_{\mathrm{HII}}=\left(3 \dot{N} / 4 \pi \alpha_{\text {rec }}\right)^{1 / 3} n_{\infty}^{-2 / 3}$ (Osterbrock \& Ferland 2006). The number of ionizing photons per unit time emitted from the innermost parts of a BH's accretion flow is

$$
\dot{N}=\int_{\nu_{0}}^{\infty} \frac{L_{\nu}}{h \nu} d \nu \approx \frac{\alpha-1}{\alpha}\left(\frac{L_{\mathrm{bol}}}{h \nu_{0}}\right)
$$

which is characterized by bolometric luminosity $L_{\mathrm{bol}}=$ $\int_{0}^{\infty} L_{\nu} d \nu$ and $L_{\nu} \propto \nu^{-\alpha}$. Here, $\alpha=1.5$ is the spectroscopic index representative of the spectral energy distribution (SED) of active galactic nuclei (AGN) in the high accretion state, and $h \nu_{0}=13.6 \mathrm{eV}$ for hydrogen. $\alpha_{\text {rec }}=4 \times 10^{-13} \mathrm{~cm}^{3} \mathrm{~s}^{-1}$ corresponds to the Case A recombination coefficient for hydrogen gas at the temperature $T_{\infty}=10^{4} \mathrm{~K}$, used here so to match simulations described in the next section. Note that the Case B coefficient is a more appropriate choice for the high gas densities in this problem, which would result in a small change Strömgren radius being proportional to $\alpha_{\text {rec }}^{-1 / 3}$. We consider accretion powered $\mathrm{BH}$ luminosity, $L_{\mathrm{bol}}=\eta \dot{M} c^{2}$, and assume radiative efficiency $\eta=0.1$ (Shakura \& Sunyaev 1973).

These ingredients provide an estimate for the size of the $\mathrm{H}$ II region, given a known $\mathrm{MBH}$ accretion rate. In the case of moving MBHs with the Mach number in the 
TABLE 1

Simulation Parameters

\begin{tabular}{ccccc}
\hline \hline ID & $N_{\mathrm{r}} \times N_{\theta}$ & $\Delta r / r$ & $\mathcal{M}$ & $t_{\text {end }}(\mathrm{Myr})$ \\
\hline HR & $400 \times 128$ & 0.026 & $0.5,1.0,2.0$ & $201.0,74.9,74.9$ \\
MR & $300 \times 96$ & 0.035 & $0.5,1.0,2.0$ & $201.0,201.0,201.0$ \\
LR & $200 \times 64$ & 0.053 & $0.5,1.0,1.5,2.0$ & $201.0,201.0,201.0,201.0$ \\
\hline
\end{tabular}

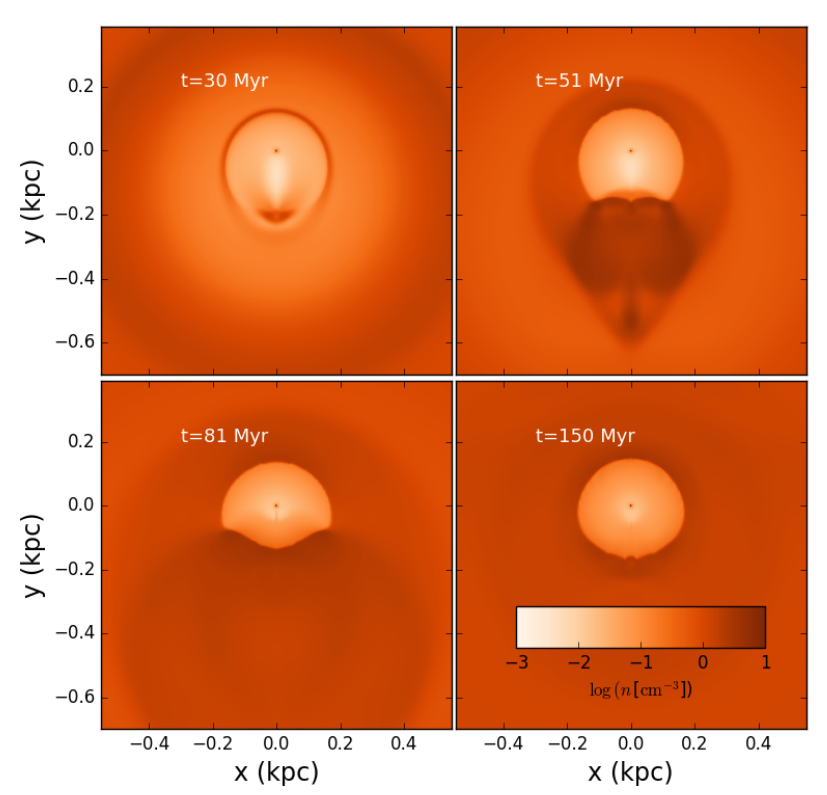

Fig. 1.- Snapshots of gas number density at different times for the HR run with $\mathcal{M}=0.5$. The flow of gas is from top to bottom and the $\mathrm{BH}$ is located at $(x, y)=(0,0)$ in each panel. The shape of the $\mathrm{H}$ II region and dynamical friction wake reach steady state after $150 \mathrm{Myr}$ in this simulation.

range $1<\mathcal{M}<4$, PR13 find that the accretion rate mediated by radiation feedback can be expressed as

$$
\dot{M} \approx 1.2 \times 10^{-2} \mathcal{M}^{2} \dot{M}_{\mathrm{B}}\left(\frac{T_{\infty}}{T_{\text {in }}}\right)^{5 / 2}
$$

where $\dot{M}_{\mathrm{B}}$ is the nominal Bondi accretion rate for isothermal gas (Bondi \& Hoyle 1944)

$$
\begin{aligned}
& \dot{M}_{\mathrm{B}}=\frac{\pi e^{3 / 2} \rho_{\infty}\left(G M_{\mathrm{BH}}\right)^{2}}{c_{s, \infty}^{3}} \\
& =8.7 \times 10^{-3}\left(\frac{n_{\infty}}{1 \mathrm{~cm}^{-3}}\right)\left(\frac{T_{\infty}}{10^{4} \mathrm{~K}}\right)^{-\frac{3}{2}}\left(\frac{M_{\mathrm{BH}}}{10^{6} M_{\odot}}\right)^{2} M_{\odot} \mathrm{yr}^{-1} .
\end{aligned}
$$

Combining Equations (3)-(5), we obtain the estimate for the radius of the $\mathrm{H}$ II sphere

$R_{\mathrm{HII}} \approx 430 \mathrm{pc} \mathcal{M}^{2 / 3}\left(\frac{n_{\infty}}{1 \mathrm{~cm}^{-3}}\right)^{-\frac{1}{3}}\left(\frac{T_{\mathrm{in}}}{4 \times 10^{4} \mathrm{~K}}\right)^{-\frac{1}{2}}\left(\frac{M_{\mathrm{BH}}}{10^{6} M_{\odot}}\right)^{\frac{2}{3}}$.

Considering the ratio of Equations (6) and (2) we get

$$
\frac{R_{\mathrm{HII}}}{R_{\mathrm{DF}}} \sim 8 \mathcal{M}^{\frac{2}{3}}\left(\frac{T_{\infty}}{10^{4} \mathrm{~K}}\right)\left(\frac{T_{\mathrm{in}}}{4 \times 10^{4} \mathrm{~K}}\right)^{-\frac{1}{2}}\left(\frac{M_{\mathrm{BH}} n_{\infty}}{10^{6} M_{\odot} \mathrm{cm}^{-3}}\right)^{-\frac{1}{3}}
$$

where we find that $R_{\mathrm{HII}} \gtrsim R_{\mathrm{DF}}$ for all values of the Mach number in the range $1<\mathcal{M}<4$ and when

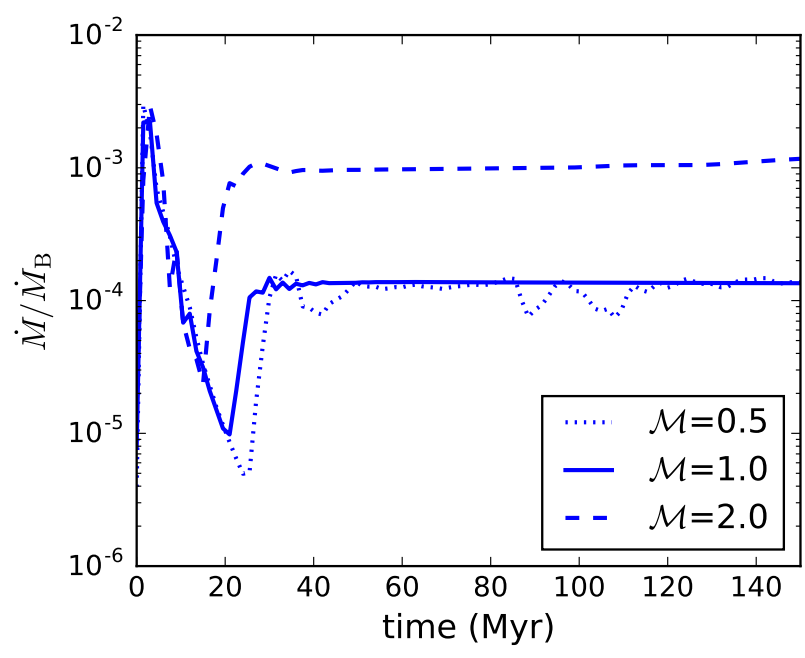

Fig. 2.- Accretion rate onto the $\mathrm{MBH}$ as a function of time shown in units of Bondi accretion rate defined in Equation (5). Different line styles mark MR runs with $\mathcal{M}=0.5,1.0$, and 2.0.

$\left(1+\mathcal{M}^{2}\right) M_{\mathrm{BH}} n_{\infty}<10^{9} M_{\odot} \mathrm{cm}^{-3}$. It follows that in this regime the dynamical friction wake is likely to be fully ionized and dispersed by the $\mathrm{MBH}$ radiative feedback, especially given the proximity of the wake mentioned before.

In the next section we examine the detailed structure of the wake and the $\mathrm{H}$ II region, and numerically evaluate the resulting dynamical friction force from radiation hydrodynamic simulations.

\section{RADIATION-HYDRODYNAMIC SIMULATIONS}

\subsection{Numerical Setup}

We run a set of $2 \mathrm{D}$ radiation hydrodynamic simulations using a parallel version of the non-relativistic code ZEUS-MP (Stone \& Norman 1992; Hayes et al. 2006). Simulations are carried out in a polar coordinate system defined by coordinates $(r, \theta)$ and assuming axisymmetry with respect to the MBH's direction of motion. The extent of the computational domain is given by $r \in(0.1 \mathrm{pc}, 3.0 \mathrm{kpc})$ and $\theta \in(0, \pi)$ and other relevant quantities are shown in Table 1 . In the calculation of gas dynamics we consider only the gravitational potential of the $\mathrm{MBH}$ and neglect the self-gravity of the gas.

An $\mathrm{MBH}$ with mass $M_{\mathrm{BH}}=10^{6} M_{\odot}$, located at the origin of the coordinate system $(r=0)$, is placed in a "wind tunnel." In this setup, the gas of uniform density $n_{\infty}=1.0 \mathrm{~cm}^{-3}$ and temperature $T_{\infty}=10^{4} \mathrm{~K}$ is assumed to flow into the computational domain in direction $\theta=$ $\pi$. We evaluate the accretion rate onto the $\mathrm{MBH}$ by calculating the mass flux through the inner boundary of the computational domain, defined by the sphere with radius $r_{\min }=0.1 \mathrm{pc}$, and convert it to $\mathrm{MBH}$ luminosity as $L_{\mathrm{bol}}=0.1 \dot{M} c^{2}$. The $\mathrm{SED}$ of ionizing radiation is described as $L_{\nu} \propto \nu^{-1.5}$ using 50 energy bins ranging 

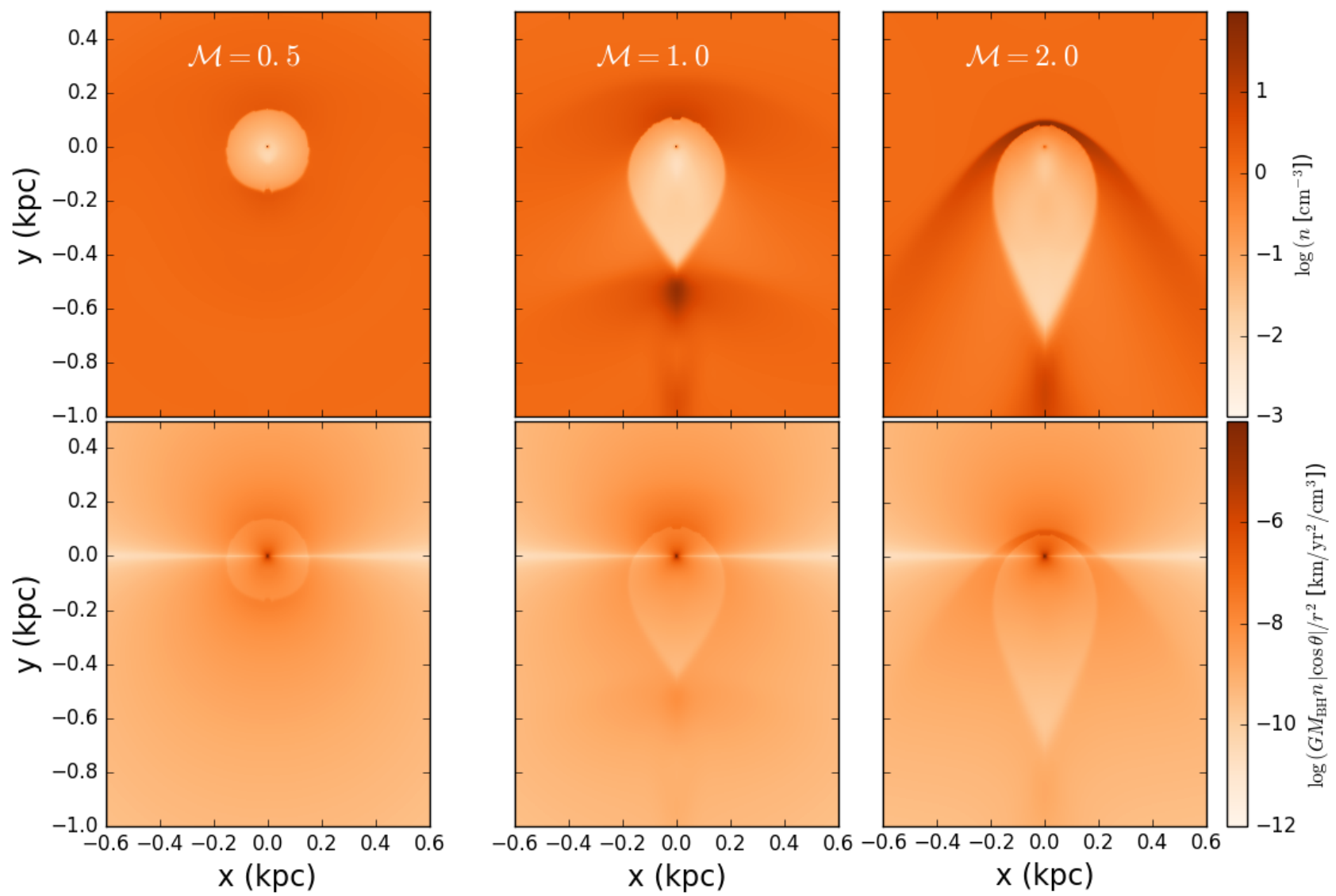

FIG. 3.- Top: 2D snapshots of the number density of gas at $\mathrm{t}=150 \mathrm{Myr}$ for $\mathrm{MR}$ runs $\mathcal{M}=0.5,1.0$, and 2.0 (left to right). All snapshots show steady state gas distributions. Bottom: contribution to dynamical friction from the gas surrounding the MBH, evaluated as the gravitational acceleration per unit volume along the direction of the MBH motion.

from $13.6 \mathrm{eV}$ to $100 \mathrm{keV}$.

The composition of the $\mathrm{H}-\mathrm{He}$ gas is evolved by following the species of Hi, HiI, HeI, HeII, HeIII, and $e^{-}$. The radiation transport is coupled with hydrodynamics and includes photo-heating, photo-ionization, Compton heating by UV and X-ray photons from the $\mathrm{BH}$, and gas cooling (Park \& Ricotti 2011, 2012; Park et al. 2014a,b). We assume that $\mathrm{BH}$ radiation is the only heating source and adopt a simple analytic form of atomic cooling function $\Lambda(T)$ due to neutral and ionized $\mathrm{H}$ and $\mathrm{He}$, which includes cooling by recombination, collisional ionization/excitation, free-free transitions, and dielectric recombination of He II (Shapiro \& Kang 1987). Molecular cooling is neglected and the cooling rate is set to zero below $T=10^{4} \mathrm{~K}$. The calculation of radiation transport accounts for the radiation pressure and thus allows us to accurately capture the effects of both energy and momentum feedback (see Park \& Ricotti 2012, for implementation details). Hydrodynamic and radiative transfer equations are solved at every time step defined as $\Delta t=\min \left(\Delta_{\text {hydro }}, \Delta_{\text {chem }}\right)$, where $\Delta_{\text {hydro }}$ is the hydrodynamical time step and $\Delta_{\text {chem }}$ is the time step required to calculate the change of chemical abundances (Ricotti et al. 2001; Whalen \& Norman 2006).

We investigate the effect of radiative feedback on the dynamical friction wake by varying the MBH Mach number from 0.5 (subsonic) to 2.0 (supersonic), while keep- ing $M_{\mathrm{BH}}, n_{\infty}$, and $T_{\infty}$ fixed. Each simulation is run for several sound crossing timescales, defined as $t_{\text {cross }}=$ $R_{\mathrm{HII}} / c_{s, \infty}$, which corresponds to $46 \mathrm{Myr}$ for the size of the H II region defined by equation (6). This ensures that the accretion rate onto the MBH and density distribution of the gas in the dynamical friction wake reach steady state. The length of each simulation is recorded as $t_{\text {end }}$ in Table 1 . We test numerical convergence with the set of high (HR), medium (MR), and low resolution (LR) runs. The HR runs are carried out with resolution of $\left(N_{r} \times N_{\theta}\right)=(400 \times 128)$, MR with $(300 \times 96)$, and LR with $(200 \times 64)$. The radial bins are logarithmically spaced so that $\Delta r / r$ is constant everywhere on the grid while the bins in polar angle are evenly spaced and have size $\Delta \theta=\pi / N_{\theta}$. The outer boundary of the computational domain is defined as inflow where $0 \leq \theta \leq \pi / 2$ and outflow for $\pi / 2<\theta \leq \pi$. We apply the outflow boundary conditions at the inner domain boundary.

\subsection{Evolution of the $\mathrm{H}$ II region and overdensity wake}

The snapshots in Figure 1 illustrate the evolution of the gas density in the $\mathrm{HR}$ run with $\mathcal{M}=0.5$. A low density $\mathrm{H}$ II region with $T_{\text {in }} \approx 6 \times 10^{4} \mathrm{~K}$ forms promptly around the $\mathrm{MBH}$, while the distribution of gas outside of it reaches steady state $150 \mathrm{Myr}$ after the beginning of the simulation. This timescale is consistent with $\sim 7$ sound crossing times for the $\mathrm{H}$ II region of the size $200 \mathrm{pc}$. 

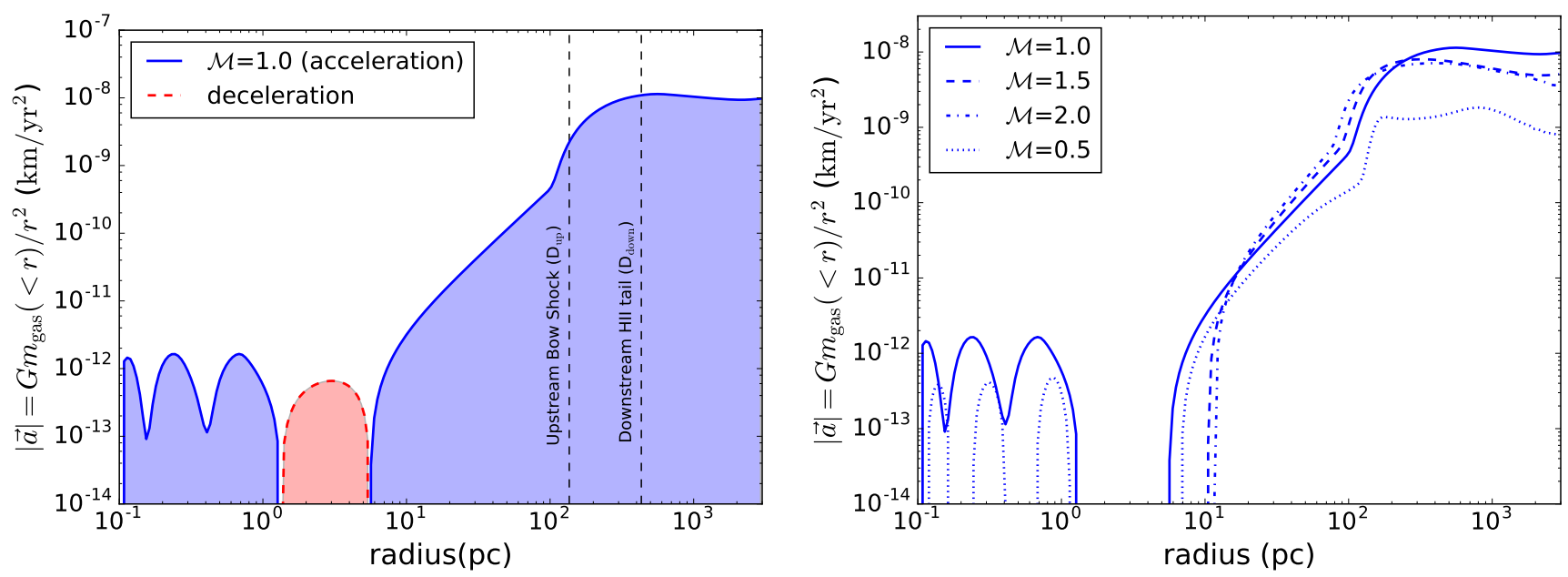

FIG. 4.- Left: magnitude of the MBH acceleration along the direction of motion (either positive or negative) contributed by the gas within polar radius $r$ for the LR $\mathcal{M}=1.0$ run. The MBH is located at the origin $r=0$. Dashed lines mark the locations of the upstream bow shock and the tail of the downstream H II region, respectively. Right: magnitude of the positive component of acceleration (resulting in $\mathrm{MBH}$ speed-up) contributed by the gas within $r$. Different lines mark LR runs with $\mathcal{M}=0.5,1.0,1.5$, and 2.0.

Once steady state is achieved, the shape of the $\mathrm{H}$ II region shows minor deviation from spherical symmetry.

Figure 2 shows the accretion rate as a function of time for the MR runs with $\mathcal{M}=0.5,1.0$, and 2.0. In all simulations $\mathrm{MBH}$ initially exhibits a relatively high accretion rate, which decreases as the $\mathrm{H}$ II region expands into the background medium as a consequence of the MBH radiative feedback. The expansion of the $\mathrm{H}$ II sphere is brought to a halt at $\sim 15-30 \mathrm{Myr}$ after the beginning of the simulation, at which point the accretion rate reaches a turning (minimum) point and readjusts to a steady state value after $\sim 20-35 \mathrm{Myr}$. This happens first in the LR run with $\mathcal{M}=2.0$, followed by $\mathcal{M}=1.0$ and 0.5 . This hierarchy of timescales is determined by the inflow rate of the gas into the $\mathrm{HII}$ region as seen by the $\mathrm{MBH}$.

Figure 2 also illustrates the dependence $\dot{M} \propto \mathcal{M}^{2}$ captured by Equation (4) and applicable in the range $1<\mathcal{M}<4$. This dependence of accretion rate on the Mach number was first reported by PR13, who noted that accretion onto the $\mathrm{MBH}$ is mediated by a dense shell, which forms upstream from the $\mathrm{MBH}$, at the interface of the $\mathrm{H}$ II region and ambient gas. This dense shell and the associated bow shock are discernible in Figure 3, in the MR runs with $\mathcal{M}=1.0$ and 2.0 , and are absent for the subsonic run with $\mathcal{M}=0.5$. The same figure shows that the $\mathrm{H}$ II region becomes more elongated in the direction of the MBH motion with the increasing Mach number, engulfing a larger volume in the region where the dynamical friction wake is supposed to form. Despite this effect, a noticeable overdensity of gas persists at the tail of the HII region in all simulations, indicating that some fraction of the dynamical friction wake remains present.

The bottom panels of Figure 3 illustrate the strength of gravitational interaction between the $\mathrm{MBH}$ and fluid elements in the computational domain, where we evaluate the magnitude of the acceleration per unit volume along the direction of the $\mathrm{BH}$ motion as $G M_{\mathrm{BH}} n|\cos \theta| / r^{2}$. The net effect of a given fluid element on the MBH depends on its location. The gas in the upstream $(0 \leq \theta \leq$ $\pi / 2)$ acts to accelerate the $\mathrm{MBH}$, while the gas in the downstream $(\pi / 2 \leq \theta \leq \pi)$ decelerates it. The shade of the color indicates that the MBH most strongly interacts with the gas in its immediate vicinity, within a radius of a few parsecs, but this distribution appears front-back symmetric and is thus not expected to significantly contribute to the acceleration of the MBH. Similarly, the fluid elements perpendicular to the MBH's line of motion at $\theta \approx \pi / 2$ make a negligible contribution. The overdensity of gas that forms at the front and tail of the $\mathrm{H}$ II region is thus expected to contribute the most to the net acceleration of the MBH. In the run with $\mathcal{M}=0.5$ this distribution is approximately front-back symmetric, indicating a lower magnitude of acceleration. The $\mathcal{M}$ $=1.0$ and 2.0 runs, on the other hand, show enhanced contribution to $\mathrm{MBH}$ acceleration from the front of the $\mathrm{H}$ II region, while contribution from the tail appears less significant. In order to determine whether the MBH accelerates or decelerates, once the steady state distribution of gas is achieved, we integrate contributions to the dynamical friction force from all fluid elements in the computational domain.

\subsection{Efficiency of dynamical friction}

The left panel of Figure 4 shows the steady state magnitude of the $\mathrm{MBH}$ acceleration (either positive or negative) due to the gas enclosed within the sphere of radius $r$ for the LR run with $\mathcal{M}=1.0$. Positive acceleration is defined to be in the direction of the $\mathrm{MBH}$ motion, thus resulting in the speed-up of the MBH. Note that the assumption of azimuthal symmetry guarantees that the components of acceleration perpendicular to the MBH's line of motion cancel out, leaving the parallel components as the only contribution.

The magnitude of acceleration is relatively low in the immediate vicinity of the MBH $(r<3 \mathrm{pc})$, consistent with the earlier observation of the front-back symmetry in this region. At $r \gtrsim 3 \mathrm{pc}$ the magnitude of acceleration gradually increases as the contrast between the upstream and downstream distribution of gas increases with radius. At $r \approx 100 \mathrm{pc}$, acceleration shows a sudden jump coinciding with the upstream overdensity and associated bow shock. The acceleration magnitude levels off 


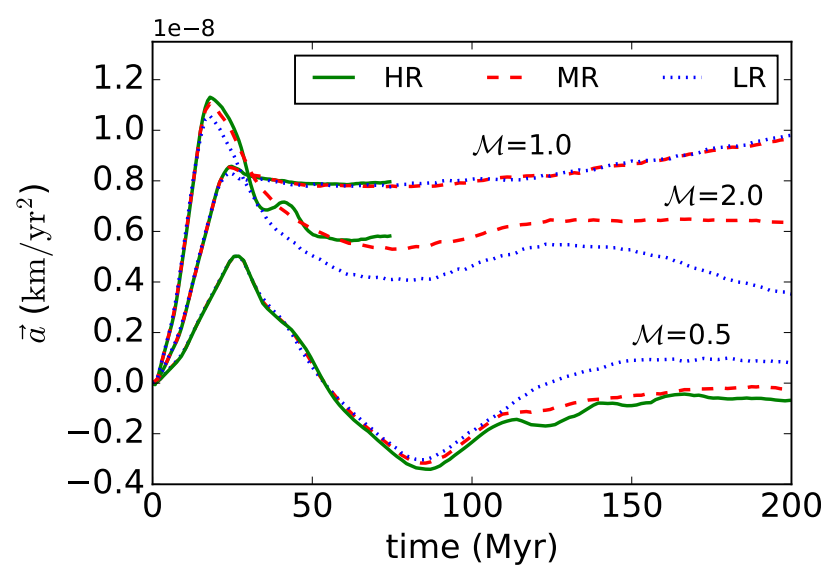

FIG. 5.- Evolution of the net acceleration, integrated over the entire computational domain, for the HR (solid lines), MR (dashed), and LR (dotted) runs with $\mathcal{M}=0.5,1.0$, and 2.0. Positive acceleration implies speed-up of the MBH.

beyond $400 \mathrm{pc}$, which marks the spatial extent of the tail of the H II sphere. Evidently, the dynamical friction wake does not contribute to the $\mathrm{MBH}$ acceleration beyond this point.

The right panel of Figure 4 shows the magnitude of the positive component of $\mathrm{MBH}$ acceleration contributed by the gas enclosed within radius $r$. The figure illustrates that in all simulations the largest contribution to the $\mathrm{MBH}$ acceleration originates from the region $r \gtrsim 10 \mathrm{pc}$. This acceleration is net positive, thus speeding up the $\mathrm{MBH}$. In all cases, the $\mathrm{MBH}$ deceleration due to the wake beyond the tail of the $\mathrm{H}$ II region is negligible. The acceleration originating from $r<10 \mathrm{pc}$ can be either positive or negative but is orders of magnitude smaller relative to the larger spatial scales and can be neglected.

Figure 5 shows the evolution of the net acceleration integrated over the entire computational domain as a function of time. In all cases acceleration initially peaks at $15-30$ Myrs. This is the same point in time where the accretion rate reaches minimum (Figure 2), indicating a buildup of the dense shell of gas in the upstream of the $\mathrm{MBH}$, which exerts gravitational influence and initially suppresses accretion onto the $\mathrm{MBH}$. Gravitational influence of the upstream gas shell is counterbalanced by the gas in the downstream of the $\mathrm{MBH}$, where overdensity wake develops with a delay corresponding to a few sound crossing times across the elongated H II region. Consequently, the $\mathrm{MBH}$ acceleration reaches steady state with a delay of $\sim 150 \mathrm{Myr}$, long after the accretion rate has settled into steady state. An inspection of Figure 5 shows that the net acceleration for the scenario $\mathcal{M}=1.0$ continues to gently increase even after $200 \mathrm{Myr}$.

For $\mathcal{M}=1.0$ and 2.0 the net end value of acceleration is positive, indicating a speed-up of the MBH. As expected, the magnitude of acceleration is smallest for the $\mathcal{M}=0.5$ scenario, where the gas flow around the $\mathrm{MBH}$ exhibits a large degree of front-back symmetry. Figure 5 illustrates that the sign of net acceleration in this case is resolution dependent, where the final outcome of the LR run is MBH speed-up and in the MR and HR runs, deceleration. The net accelerations in the $\mathrm{MR}$ and HR runs track each other closely and differ by only $\sim 0.05 \mathrm{~km} \mathrm{yr}^{-2}$, indicating numerical convergence of the $\mathrm{MR}$ run. We therefore use this value as a measure

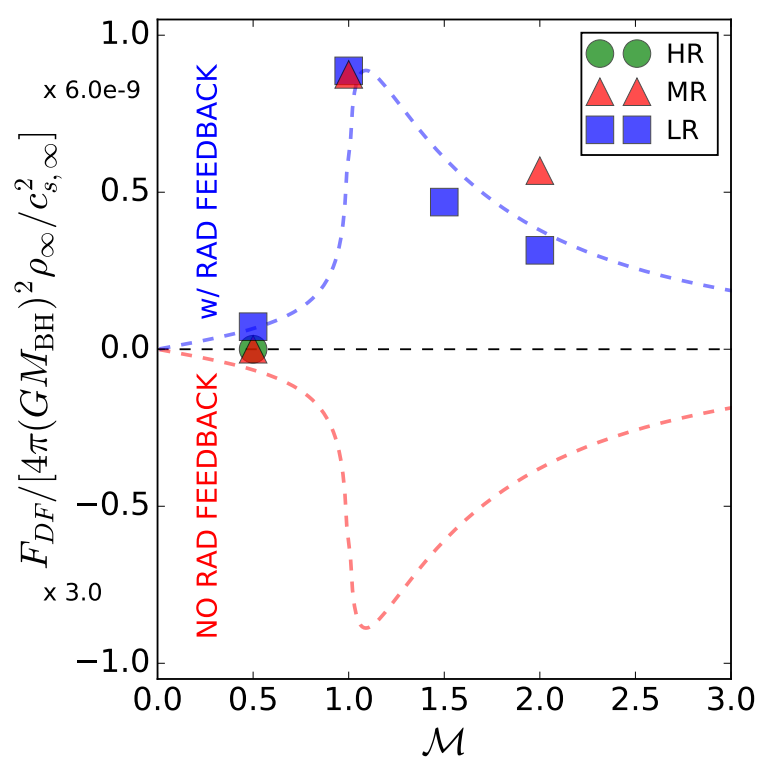

FIG. 6.- Dynamical friction force as a function of the Mach number. The red dashed line illustrates the magnitude of the dynamical friction force in the absence of radiative feedback as calculated by Ostriker (1999). The blue dashed line is the same as the red one but plotted with a positive sign and arbitrarily scaled in magnitude to match data points. Different symbols mark results of the runs with radiative feedback at $t=201.0 \mathrm{Myr}$. Note the different magnitudes of the positive and negative $y$-axis.

of error in net acceleration due to the finite numerical resolution of our simulations.

Figure 6 shows the values of the dynamical friction force measured at the end of each simulation for different values of $\mathcal{M}$. As a comparison, the red dashed line shows the dynamical friction force in the absence of radiative feedback, as predicted by Ostriker (1999) for $\ln \left(c_{s, \infty} t / r_{\min }\right)=4$. As noted before, the dynamical friction force calculated from simulations presented in this study is net positive for $1.0 \leq \mathcal{M}<4$ and negative for $\mathcal{M}=0.5$. The magnitude of this force is however over $\sim 9$ orders of magnitude lower compared to that experienced by the MBHs in absence of radiative feedback and is therefore negligible for all values of the Mach number $\mathcal{M}<4$. Interestingly, the dynamical friction force in the presence of radiative feedback appears to mirror its classical counterpart: it peaks around $\mathcal{M} \approx 1$ and decreases at larger values of the Mach number.

\section{DISCUSSION AND CONCLUSIONS}

We investigate the efficiency of dynamical friction in presence of radiative feedback from an $\mathrm{MBH}$ moving through a uniform density gas. Ionizing radiation that emerges from the innermost parts of the MBH's accretion flow results in the formation of the $\mathrm{H}$ II region, which strongly affects the dynamical friction wake and renders dynamical friction inefficient for a range of physical scenarios. We summarize our main findings below:

- We identify a physical regime in which the dynamical friction wake is likely to be fully ionized and dispersed by the $\mathrm{MBH}$ radiative feedback as a regime in which the radius of the $\mathrm{H}$ II sphere is larger than the extent of the dynamical friction wake. This condition is fulfilled when $\mathcal{M}<4$ 
and $\left(1+\mathcal{M}^{2}\right) M_{\mathrm{BH}} n_{\infty}<10^{9} M_{\odot} \mathrm{cm}^{-3}$. Outside of this regime the formation of the H II region is suppressed and the properties of the gas flow around the $\mathrm{MBH}$ are restored to the classical Bondi-HoyleLyttleton solution, thus restoring the effect of dynamical friction. These criteria can be utilized as a sub-resolution model for dynamical friction in large scale simulations that do not resolve the scale of the $\mathrm{H}$ II region or $\mathrm{MBH}$ density wake.

- Based on radiation hydrodynamic simulations we find that the net acceleration experienced by the $\mathrm{MBH}$ in this regime tends to be positive, meaning that they speed up, contrary to the expectations for gaseous dynamical friction in absence of radiative feedback. This reversal happens because the dominant contribution to the $\mathrm{MBH}$ acceleration comes from the dense shell of gas that forms in front of the $\mathrm{MBH}$ as a consequence of the snowplow effect caused by radiation pressure.

- The magnitude of $\mathrm{MBH}$ acceleration peaks at $\mathcal{M} \approx$ 1 and decreases for larger values of the Mach number, similar to the dynamical friction force in absence of radiative feedback. The magnitude of acceleration is however negligibly small, implying that $\mathrm{MBH}$ in this regime will not significantly change their velocity over timescales that determine their motion and properties of their environment.

- Our results suggest that suppression of dynamical friction by radiative feedback should be more severe at the lower mass end of the $\mathrm{MBH}$ spectrum, because these BHs must reside in regions of relatively high gas density in order to experience efficient dynamical friction in the regime when $\left(1+\mathcal{M}^{2}\right) M_{\mathrm{BH}} n_{\infty}>10^{9} M_{\odot} \mathrm{cm}^{-3}$. Compounded with inefficiency of the gas drag for lower mass objects in general this implies that $<10^{7} M_{\odot}$ MBHs have fewer means to reach the centers of merged galaxies.

This study makes several idealized assumptions, such as the uniform gas density and infinite background medium. In reality however, MBHs in the aftermath of galactic mergers are likely to find themselves immersed in inhomogeneous and clumpy medium which can perturb their otherwise smooth orbital decay (Fiacconi et al. 2013). Furthermore, the geometry and properties of the $\mathrm{H}$ II region will be affected in scenarios where the $\mathrm{H}$ II region is not confined by the gaseous medium. An example of this would be an $\mathrm{H}$ II region around the $\mathrm{MBH}$ orbiting within the galactic gas disk. When the radius of the $\mathrm{H}$ II region exceeds the half thickness of the disk, the radiation can escape outside of the disk plane, making the problem fully three-dimensional. Even in such scenarios, the proximity of the dynamical friction wake to the $\mathrm{MBH}$ is likely to result in the wake obliteration by high energy radiation. Indeed, 3D hydrodynamic simulations that capture the dynamics of MBHs in proper galactic setups also find a weakening of the dynamical friction force in the presence of MBH feedback (Sijacki et al. 2011; Souza Lima et al. 2016). The high resolution, local simulations presented here support these findings and provide a set of criteria, formulated in terms of the properties of the gas and $\mathrm{MBH}$, under which the wake evacuation is efficient.

Along similar lines, we consider an isolated $\mathrm{MBH}$ on a linear trajectory, whereas the front-back asymmetry of the density wake for a perturber on a circular orbit has been shown to cause small differences in the dynamical friction force in absence of radiative feedback (Kim \& Kim 2007). A system consisting of multiple MBHs would further add to the complexity in cases when dynamical friction wakes can mutually affect one another (e.g., Kim et al. 2008). This type of study may require a transition from $2 \mathrm{D}$ to $3 \mathrm{D}$ simulations in the future in order to relax the assumption of azimuthal symmetry and properly capture the interplay of inhomogeneities, radiative feedback, and dynamical friction.

This work is supported in part by the National Science Foundation under the Theoretical and Computational Astrophysics Network (TCAN) grant AST1333360. T.B. acknowledges support from the Research Corporation for Science Advancement through a Cottrell Scholar Award. T.B. is a member of the MAGNA project (http://www.issibern.ch/teams/agnactivity) supported by the International Space Science Institute (ISSI) in Bern, Switzerland. Numerical simulations presented in this paper were performed using the high-performance computing cluster PACE, administered by the Office of Information and Technology at the Georgia Institute of Technology.

\section{REFERENCES}

Begelman, M. C. 1978, MNRAS, 184, 53

-. 1979, MNRAS, 187, 237

Bondi, H., \& Hoyle, F. 1944, MNRAS, 104, 273

Callegari, S., Kazantzidis, S., Mayer, L., et al. 2011, ApJ, 729, 85

Callegari, S., Mayer, L., Kazantzidis, S., et al. 2009, ApJ, 696, L89

Chandrasekhar, S. 1943, ApJ, 97, 255

Chapon, D., Mayer, L., \& Teyssier, R. 2013, MNRAS, 429, 3114

Dotti, M., Colpi, M., \& Haardt, F. 2006, MNRAS, 367, 103

Escala, A., Larson, R. B., Coppi, P. S., \& Mardones, D. 2005, ApJ, 630, 152

Fiacconi, D., Mayer, L., Roškar, R., \& Colpi, M. 2013, ApJ, 777, L14

Hayes, J. C., Norman, M. L., Fiedler, R. A., et al. 2006, ApJS, 165,188

Hoyle, F., \& Lyttleton, R. A. 1939, in Proceedings of the Cambridge Philosophical Society, Vol. 35, Proceedings of the Cambridge Philosophical Society, 405-+
Inayoshi, K., Haiman, Z., \& Ostriker, J. P. 2016, MNRAS, 459, 3738

Kim, H., \& Kim, W.-T. 2007, ApJ, 665, 432

Kim, H., Kim, W.-T., \& Sánchez-Salcedo, F. J. 2008, ApJ, 679, L33

Li, Y. 2011, ArXiv e-prints, arXiv:1109.3442

Mayer, L. 2013, Classical and Quantum Gravity, 30, 244008

Mayer, L., Kazantzidis, S., Madau, P., et al. 2007, Science, 316, 1874

Milosavljević, M., Couch, S. M., \& Bromm, V. 2009, ApJ, 696, L146

Osterbrock, D. E., \& Ferland, G. J. 2006, Astrophysics of gaseous nebulae and active galactic nuclei

Ostriker, E. C. 1999, ApJ, 513, 252

Pacucci, F., \& Ferrara, A. 2015, MNRAS, 448, 104

Park, K., \& Ricotti, M. 2011, ApJ, 739, 2

—. 2012, ApJ, 747, 9 
-. 2013, ApJ, 767, 163

Park, K., Ricotti, M., Di Matteo, T., \& Reynolds, C. S. 2014a, MNRAS, 437, 2856

-. 2014b, MNRAS, 445, 2325

Park, K., Ricotti, M., Natarajan, P., Bogdanović, T., \& Wise, J. H. 2016, ApJ, 818, 184

Ricotti, M., Gnedin, N. Y., \& Shull, J. M. 2001, ApJ, 560, 580

Shakura, N. I., \& Sunyaev, R. A. 1973, A\&A, 24, 337

Shapiro, P. R., \& Kang, H. 1987, ApJ, 318, 32
Sijacki, D., Springel, V., \& Haehnelt, M. G. 2011, MNRAS, 414, 3656

Souza Lima, R., Mayer, L., Capelo, P. R., \& Bellovary, J. M. 2016, ArXiv e-prints, arXiv: 1610.01600

Stone, J. M., \& Norman, M. L. 1992, ApJS, 80, 753

Whalen, D., \& Norman, M. L. 2006, ApJS, 162, 281 
Draft Version March 25, 2020

Typeset using $\mathrm{LATEX}_{\mathrm{E}}$ default style in AASTeX62

\author{
Erratum: "Gaseous Dynamical Friction in Presence of Black Hole Radiative Feedback" (2017, ApJ, 838, 103) \\ KWANGHo PARK ${ }^{1}$ AND TAMARA Bogdanović ${ }^{1}$ \\ ${ }^{1}$ Center for Relativistic Astrophysics, School of Physics, Georgia Institute of Technology, Atlanta, GA 30332, USA
}

(Received 2019 June 7; Revised 2019 August 19; Published 2019 October 4)

Submitted to ApJ

While performing a conversion between the units of length we inadvertently introduced an error in the figures that show the magnitude of the acceleration (and by relation, force) experienced by the MBH due to gravitational interactions with the surrounding gas. Specifically, the error occurred in the conversion to the unit of "km" in $\mathrm{km}^{-2}$ in the acceleration, where instead of converting $(\mathrm{cm} \rightarrow \mathrm{km})$, we introduced a conversion $(\mathrm{km} \rightarrow \mathrm{pc})$. As a consequence, the magnitude of the acceleration and dynamical friction force were underestimated by $3.08 \times 10^{8}$ in the figures and related text in the published article. The correction results in scaling-up of the color bar for the bottom panels of Figure 3 by a constant factor of $3.08 \times 10^{8}$. The same correction is applied to the magnitudes on the $y$-axis of Figures 4 , 5 , and 6 . The shapes of the curves in these figures, however, remain unchanged. The following bulleted list provides modifications to the specific sentences in the text, resulting from the above correction.

1. Abstract. The fifth and sixth sentences should read: "The magnitude of this acceleration is $\sim 60 \%$ of the classical value in the absence of radiative feedback, implying that MBHs in this regime may accelerate significantly over timescales that determine their motion and properties of their environment. Our results suggest that MBHs at the lower-mass end of the spectrum are more likely to find themselves in the negative dynamical friction regime, which, compounded with inefficiency of the gas drag for lower mass objects in general, implies that $<10^{7} M_{\odot}$ MBHs have fewer means to reach the centers of merged galaxies."

2. Section 3.3. The second to last sentence in the fifth paragraph should read: "The net accelerations in the MR and HR runs track each other closely and differ by only $\sim 0.2 \mathrm{~km} \mathrm{yr}^{-2}$, indicating numerical convergence of the MR run." This value was obtained by multiplying the difference of $\sim 5 \times 10^{-10} \mathrm{~km} \mathrm{yr}^{-2}$, reported in Figure 5 of the published article, by a factor of $3.08 \times 10^{8}$.

3. Section 3.3. The second to last sentence of the section should read: "The magnitude of this force corresponds to about $60 \%$ of that experienced by the $\mathrm{MBH}$ in the absence of radiative feedback and is therefore significant for all values of the Mach number $\mathcal{M}<4$."

4. Section 4. The second sentence in the third bullet point should read: "The magnitude of acceleration corresponds to about $60 \%$ of that experienced by the MBHs in the absence of radiative feedback, but the sign is opposite, implying that MBHs in this regime may accelerate significantly over timescales that determine their motion and properties of their environment."

In the remainder of the text we consider the implications for the $\mathrm{MBH}$ dynamics of the acceleration caused by negative dynamical friction. Fig. 5 shows that in the setup used in our simulations the MBHs moving through the background gas with the initial Mach number of $\mathcal{M}=1-2$ experience an average acceleration of $\sim 2 \mathrm{~km} \mathrm{yr}^{-2}$ on the timescale of about $100 \mathrm{Myr}$. This timescale approximately corresponds to a few crossing times of the H II region, defined as $t_{\text {cross }}=R_{\mathrm{H} \text { II }} / c_{s, \infty}$ in the published article. In the absence of any other external forces on the MBH, this magnitude of acceleration results in a velocity increase of $\sim 7 \mathrm{~km} \mathrm{~s}^{-1}$, on top of the initial $\mathrm{MBH}$ velocity of about $10 \mathrm{kms}$.

kwangho.park@physics.gatech.edu,tamarab@gatech.edu 
The corresponding increase in the kinetic energy of the MBH over the same period of time is $\Delta \mathrm{KE} \sim 2 \times 10^{51} \mathrm{erg}$, which we compare to the energy emitted in radiation from the innermost accretion flow surrounding the MBH. This comparison is relevant because the MBH-driven radiative feedback is the main cause of the negative dynamical friction, in the regime in which it strongly modifies the kinematics and distribution of the surrounding gas. Namely, the energy emitted in radiation, $E_{\mathrm{rad}}=0.1 \dot{M} c^{2} \times 100 \mathrm{Myr} \approx 1.6 \times 10^{55} \mathrm{erg}$, where the accretion rate $\dot{M} \approx 10^{-4} \dot{M}_{\mathrm{B}}$ is measured from simulations and shown in Figure 2 of the published article (see also Equations (4) and (5) in the published article for dependence of $\dot{M}$ on physical properties of the system). Therefore, the acquired kinetic energy of the MBH is only a small fraction of the radiated energy. Just recently, Gruzinov et al. (2020) also reported their analysis of negative dynamical friction on compact objects with powerful outflows moving through a dense gaseous medium. Based on analytic considerations they also conclude that conservation laws in principle allow negative total friction.

It is worth noting that negative dynamical friction is a self-limiting process, and the $\mathrm{MBH}$ is not expected to perpetually accelerate. As the $\mathrm{MBH}$ speeds up (i.e., with increasing $\mathcal{M}$ ), the dynamical friction force weakens, as shown in Fig. 6. Moreover, a fundamental limit exists for the maximum MBH velocity that can be achieved as a consequence of negative dynamical friction. Specifically, the jump conditions for ionization fronts predict that there is a maximum $\mathrm{MBH}$ velocity beyond which a dense ionization front ceases to exist. This occurs when the velocity reaches $\mathcal{M}>2 c_{\mathrm{s}, \text { in }} / c_{\mathrm{s}, \infty} \approx 4$, where $c_{\mathrm{s}, \text { in }}$ and $c_{\mathrm{s}, \infty}$ are the sound speed inside the ionized region and at "infinity," where it is not affected by the MBH's gravity or radiation, respectively. Detailed simulations of ionization fronts created by moving MBHs powered by accretion confirm this expectation and show that beyond this threshold the dense (a.k.a., $D$-type) ionization front transforms into the so-called rarefied ( $R$-type) ionization front (see Park \& Ricotti 2013, for a more nuanced analysis of this effect). The dissipation of the dense front leading the MBH on its trajectory results in the recovery of the Bondi-Hoyle-Lyttleton flow around the MBH. This transition most likely restores the classical dynamical friction, leading to deceleration of the MBH. Taken at the face value, and in the absence of any other external forces, this fundamental limit suggests that MBHs that are subject to negative dynamical friction should move with an equilibrium value of the Mach number of a few. There are, however, other phenomena in real galaxies that may influence the dynamical evolution of MBHs and change this outcome. MBHs in the aftermath of galactic mergers are likely to find themselves on curved orbits, immersed in inhomogeneous, clumpy, and magnetized media, which can perturb their otherwise smooth orbital evolution. On timescales considered here $\left(\sim 10^{8} \mathrm{yr}\right)$ moving MBHs are also likely to experience significant interactions with the stars in their host galaxies. The net impact of all these effects on the dynamics and evolution of MBHs should be carefully considered in future works.

In summary, the conclusion of our work that ionizing radiation that emerges from the innermost parts of the MBH's accretion flow strongly affects the dynamical friction wake for a range of physical scenarios remains unchanged (see the published article for formulation of criteria that describe this range). The correction to the magnitude of acceleration, however, implies that the dynamical friction force for MBHs that find themselves in this regime is comparable in magnitude but opposite in sign to the classical result, calculated in the absence of radiative feedback. Namely, MBHs in this regime tend to experience positive net acceleration, meaning that they speed up, contrary to the expectations for gaseous dynamical friction in the absence of radiative feedback. Our results suggest that this effect may be more prevalent at the lower-mass end of the MBH spectrum, implying that $<10^{7} M_{\odot}$ MBHs have fewer means to reach the centers of merged galaxies. This has important implications for the efficiency of pairing of MBHs in the aftermath of galactic mergers and may lead to suppression in the rate of coalescence of MBHs.

We would like to thank Daisuke Toyouchi for bringing up the discrepancy in the calculation to our attention. 

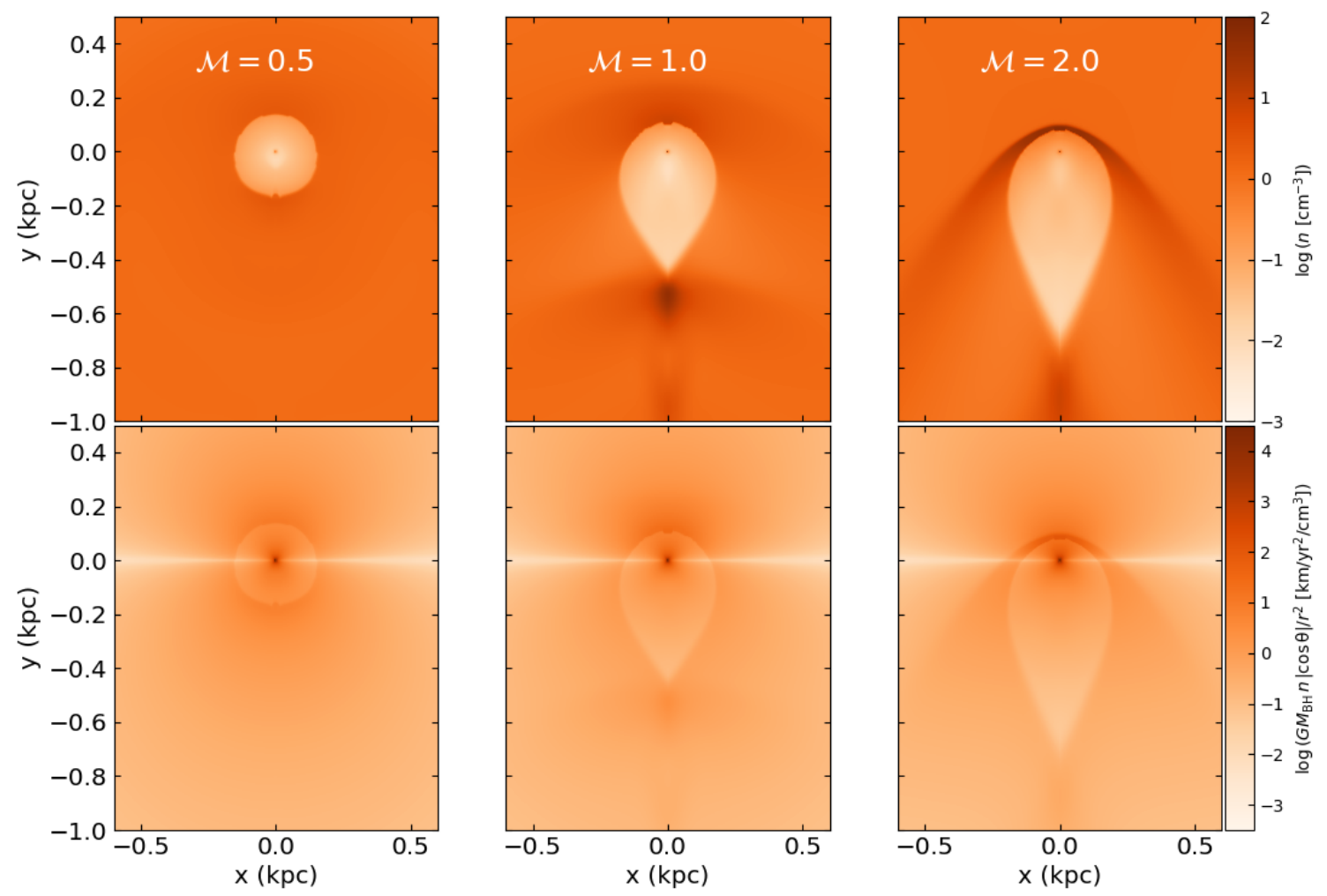

Figure 3. Top: $2 \mathrm{D}$ snapshots of the number density of gas at $t=150 \mathrm{Myr}$ for $\mathrm{MR}$ runs $\mathcal{M}=0.5,1.0$, and 2.0 (left to right). All snapshots show steady state gas distributions. Bottom: contribution to dynamical friction from the gas surrounding the $\mathrm{MBH}$, evaluated as the gravitational acceleration per unit volume along the direction of the MBH motion. (The magnitude of the acceleration per unit volume on the bottom color bar has been corrected.)
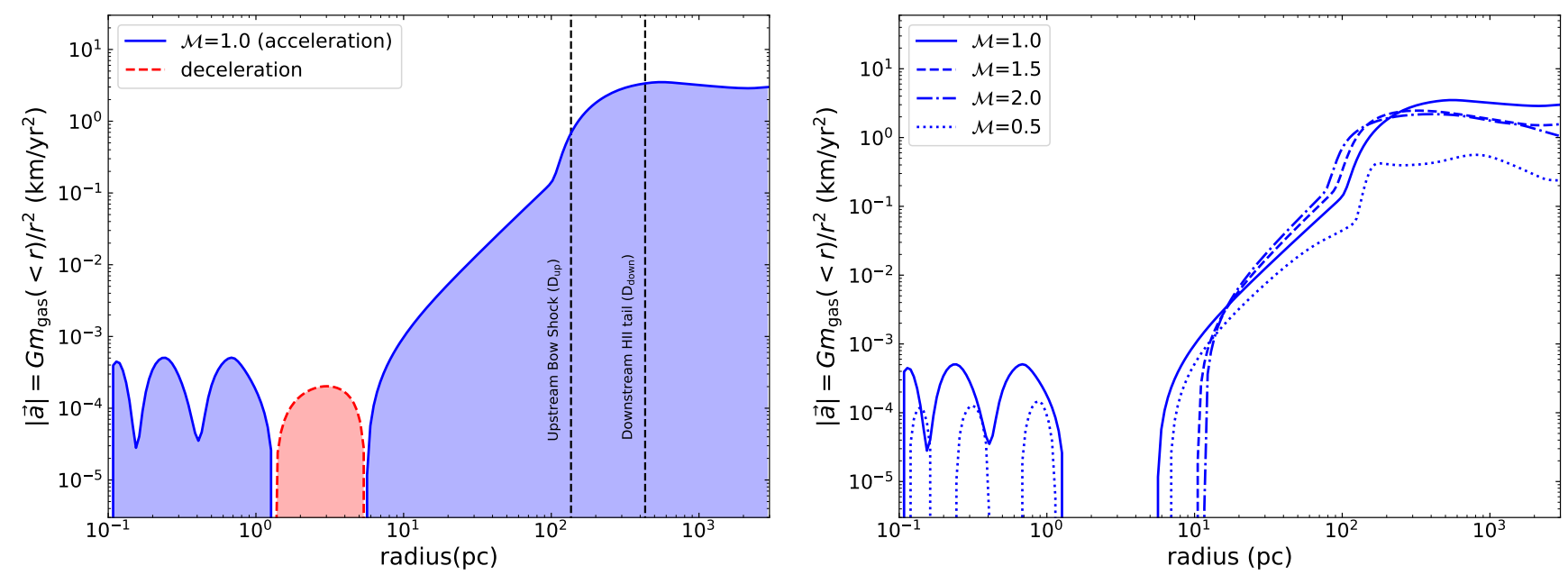

Figure 4. Left: magnitude of the $\mathrm{MBH}$ acceleration along the direction of motion (either positive or negative) contributed by the gas within polar radius $r$ for the LR $\mathcal{M}=1.0 \mathrm{run}$. The $\mathrm{MBH}$ is located at the origin $(r=0)$. Vertical dashed lines mark the locations of the upstream bow shock and the tail of the downstream H II region, respectively. Right: magnitude of the positive component of acceleration (resulting in $\mathrm{MBH}$ speed-up) contributed by the gas within $r$. Different lines mark LR runs with $\mathcal{M}$ $=0.5,1.0,1.5$, and 2.0. (The magnitude of the acceleration has been corrected in both panels.) 


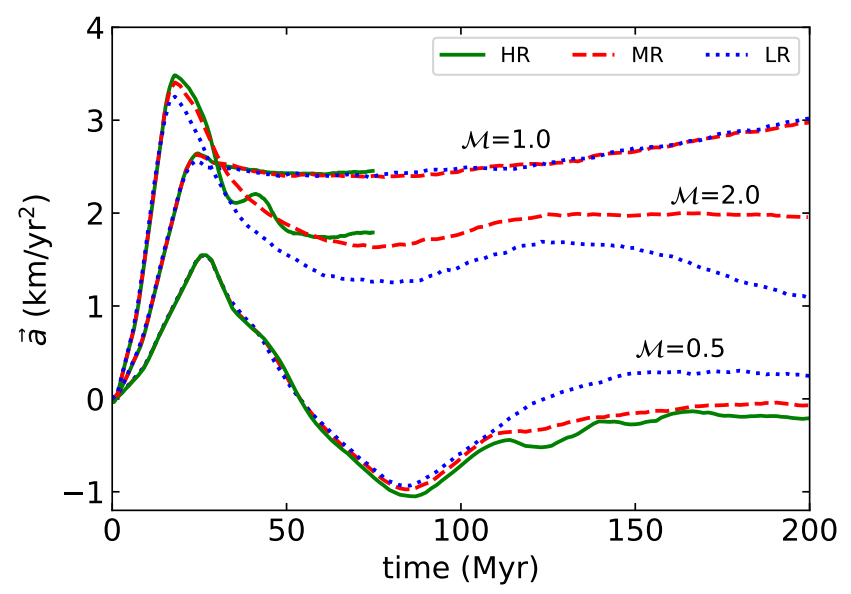

Figure 5. Evolution of the net acceleration, integrated over the entire computational domain, for the HR (solid lines), MR (dashed), and LR (dotted) runs with $\mathcal{M}=0.5,1.0$, and 2.0. Positive acceleration implies speed-up of the MBH. (The magnitude of the acceleration has been corrected.)

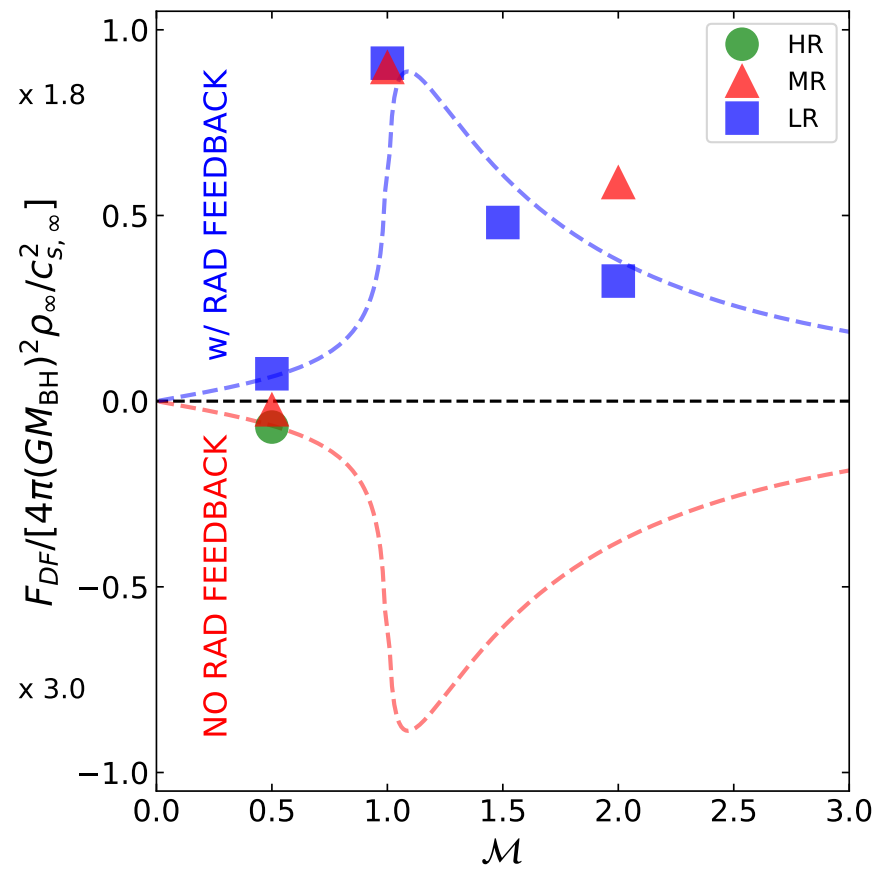

Figure 6. Dynamical friction force as a function of the Mach number. The red dashed line illustrates the magnitude of the dynamical friction force in the absence of radiative feedback as calculated by Ostriker (1999). The blue dashed line corresponds to the red one but is plotted with a positive sign (implying speed-up) and is scaled to $60 \%$ in magnitude to match data points. Different symbols mark results of the runs with radiative feedback at $t=201.0 \mathrm{Myr}$. (The magnitude of the dynamical friction force has been corrected.)

\section{REFERENCES}

Gruzinov, A., Levin, Y., \& Matzner, C. D. 2020, MNRAS,

492, 2755, doi: 10.1093/mnras/staa013
Ostriker, E. C. 1999, ApJ, 513, 252, doi: 10.1086/306858

Park, K., \& Ricotti, M. 2013, ApJ, 767, 163, doi: 10.1088/0004-637X/767/2/163 